\title{
Fenómenos del contacto lingüístico observados en quechua-hablantes en Patagonia
}

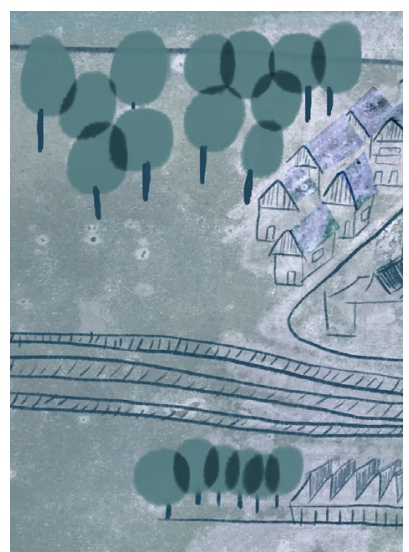

Antonio Díaz-Fernández

CELLPA / Universidad Nacional de la Patagonia San Juan Bosco, Argentina utibaitina@gmail.com

\author{
María Inés Quevedo \\ Universidad Nacional de la Patagonia San Juan Bosco, Universidad Nacional de la \\ Patagonia Austral, Argentina \\ inesquevedo@yahoo.com.ar
}

\section{Resumen}

En este trabajo se consideran las transferencias originadas por el contacto lingüístico español-quechua en vernáculohablantes residentes en Chubut, especialmente en el área del golfo San Jorge. Se analizan las ocurrencias de préstamos permanentes, o sea adaptados al sistema quechua, los préstamos momentáneos, transferencias de sintaxis e instancias de cambio de código. En estos fenómenos se observan las estrategias que el vernáculohablante utiliza en su praxis discursiva. Se identifica qué clases de palabras son las más frecuentes como préstamos, la alternancia entre transferencia y lexema vernáculo. Se identifican rasgos en el cambio de código. Asimismo, estos fenómenos estudiados permiten reflexionar sobre la competencia del hablante, es decir, el conocimiento de la gramática interna de ambos sistemas que se ponen en contacto. Como conclusión, queda claro que estos fenómenos se han incrementado debido al contacto lingüístico y a la competencia del vernáculohablante, que hace posible la ocurrencia de estos y que no muestran ningún tipo de agramaticalidad.

\section{Linguist contact features observed in Quechua-speakers living in Patagonia}

\begin{abstract}
This paper deals with transferences due to the linguistic contact between Quechua and Spanish as observed in the speech of vernacular speakers who live in Chubut, especially on the Gulf of San Jorge area. Cases of permanent loanwords, i.e. those adapted to Quechua linguistic system, nonce borrowings, syntactic transferency and cases of code switching are also
\end{abstract}

Palabras clave

quechua bilingüismo contacto lingüístico transferencias cambio de código

Keywords

quechua bilingualism language contact loan words code switching 
included in this survey. Through the use of these phenomena it is possible to see speech strategies used by the native speaker. It is also taken into account the word classes that are most frequently included as loanwords as well as the cases of alternation between vernacular words and those incorporated as loans. Finally, certain features in the use of code switching are also treated. Besides, the survey of these phenomena leads to a reflexion on the speaker's competence, i.e. the knowledge of the grammar of both systems that he puts into contact. As a conclusion, it is clear that these phenomena have increased a lot due to the linguistic contact and that the competence of vernacular speakers make possible the occurrence of these phenomena, that do not show any lack of grammaticality.

\section{Fenômenos lingüísticos de contato observados em falantes de quechua na Patagônia}

\section{Resumo}

Neste artigo, consideram-se as transferências originadas pelo contato linguístico espanhol-quechua, nos falantes vernáculos que residem na província de Chubut, especialmente na área do golfo San Jorge. Analisam-se as ocorrências de empréstimos permanentes, ou seja, adaptados ao sistema da língua quechua, os empréstimos momentâneos e as ocorrências de mudanças de código. Nestes três fenômenos constatam-se as estratégias que o falante vernáculo utiliza na sua praxis discursiva. Identifica-se que classes de palavras são as mais frequentes como empréstimos, a alternância entre a transferência e o lexema vernáculo. Observam-se traços nas mudanças de código que permitam ver sua natureza. Conclui-se que a competência do falante na língua vernácula faz possível estes fenômenos, que de modo algum resultam agramaticais.

\section{Introducción}

En este trabajo se analizan fenómenos del contacto quechua-español en hablantes bilingües en la Patagonia central, específicamente, en áreas del Golfo San Matías y Golfo San Jorge y en el sector cordillerano noroeste de la provincia de Chubut. Son migrantes del Estado Plurinacional de Bolivia, con una trayectoria de más de 10 años. Los mismos llegaron hablando ambas lenguas: el quechua como lengua materna y el español como lengua de contacto con la sociedad mayoritaria. Hoy, en su destino de migración, el uso del quechua está destinado sólo al ámbito familiar y social entre hablantes vernáculos, y no con sus hijos, a quienes no se les trasmite el uso del quechua. Mientras que el español, su segunda lengua de contacto social, es la lengua en la que han educado a sus hijos.

Se analiza el contacto quechua español en hablantes bilingües para mostrar algunas de las competencias lingüísticas que el hablante pone en juego: no solo su conocimiento léxico, sino, en el caso particular de las palabras con significado funcional, el conocimiento de los niveles morfosintáctico de ambas gramáticas. Se consideran dos fenómenos muy recurrentes y conspicuos del contacto lingüístico: el préstamo y el cambio de código. Se identifica qué clases de palabras ocurren con más frecuencia como préstamos y la alternancia entre la transferencia y el lexema vernáculo. Se observa que, si bien la influencia del español como lengua de prestigio puede dar
Palavras-chave

língua quechua bilinguismo contato linguístico transferências mudança de código 
razones contextuales para explicar el cambio, incluso en la sintaxis, es la competencia del hablante quechua la que posibilita la resolución adecuada para combinar elementos léxicos e incluso funcionales como conjunciones y preposiciones propias de una lengua flexiva en un sistema gramatical aglutinante, sin que estos fenómenos provoquen agramaticalidades.

Los fenómenos estudiados permiten reflexionar sobre la competencia del hablante, es decir, el conocimiento de la gramática interna de ambos sistemas que se ponen en contacto. Se asume que estas selecciones, que el hablante realiza, son posibles en virtud de estructuras mentales constituidas por principios generales automatizados que prescriben el funcionamiento de la facultad del lenguaje. Estos principios comunes a todos las lenguas le permiten al hablante, según su interpretación empírica de los datos que disponga, articular las condiciones formales entre diferentes gramáticas para la elección de préstamos, transferencias, o cambios de código. En este caso el hablante evalúa de manera casi automatizada combinar elementos de una lengua, el español, según las reglas del quechua. Más allá de los factores contextuales, en el caso particular de estos idiomas, influye en el contacto la situación de prestigio del castellano en cuanto al contexto histórico de la conquista y colonización de América, y en cuantoa la amplia difusión actual del español como lengua internacional frente a la reducida extensión geográfica del quechua. Estas importantes motivaciones no bastan para explicar los fenómenos del contacto en la actuación concreta de los hablantes bilingües. Es, también, el desarrollo de la competencia bilingüe, como parte de su facultad del lenguaje, lo que les permite disponer de diversos recursos lingüísticos que les ofrecen las lenguas en contacto, para realizar en su discurso la combinación de elementos de diferentes códigos lingüísticos que de ningún modo resultan agramaticales pero que sin duda responden a su necesidad de comunicación con el medio que lo rodea.

\subsection{El quechua}

El quechua es una de las familias lingüísticas sudamericanas más conocidas y más estudiadas, que cuenta con una prolífica bibliografía. Su vasto territorio se extiende desde el sur colombiano al noroeste argentino. Comprende varios grupos dialectales no siempre inteligibles entre sí. En realidad, muchos de los llamados dialectos son más bien lenguas diferentes originadas de un mismo tronco, a las cuales se las designa con el mismo glotónimo. Cerrón Palomino (1987) presenta una clasificación interna de esta familia, a la que asigna dos taxones principales: (a) la rama Waywash, con los grupos Huylay, Ap-Am-H y Huancay; y, (b) la rama Wampuy, que incluye las subramas Yungay, Chinchay norte y Chinchay sur. Dentro de esta última se incluyen las hablas de Bolivia.

Respecto a su filiación genética, la clasificación de los autores puede presentar diferencias, pero en este artículo se toma la que presenta Ruhlen (1991). El o los idiomas quechuas son incluidos en la rama Quechumara, taxón que se inserta dentro del stock Andes en el phylum Amerindio (Ruhlen 1991, 239-240).

El contacto del quechua con el español ha puesto en relación dos lenguas que presentan diferencias tipológicas muy divergentes en cuanto al funcionamiento de los sistemas morfosintácticos y otros aspectos semánticopragmáticos. El español, lengua romance, se caracteriza por el orden de palabra SVO, además de ser un idioma flexivo. Por su parte, el quechua 
tiene un orden prototípico SOV y es una lengua del tipo aglutinante. Cerrón Palomino, al referirse a la sintaxis del quechua señala lo siguiente:

el quechumara presenta un orden favorito de los elementos constitutivos mayores de aquélla, en virtud de la cual el Sujeto ( $\mathrm{A}$ o S) va delante, siguiendo el Objeto (O) y luego, cerrando el enunciado, el Verbo (V). Abreviando, se trata, pues, de un orden SOV (Cerrón Palomino 2008, 183).

Asimismo, expresa que SOV es el orden "natural y espontáneo de toda oración básica del quechumara (y ciertamente libre de toda influencia castellana)" (Cerrón Palomino 2008, 183). Pero factores pragmáticos y comunicativos pueden alterar ese orden, presentando OSV y OVS; no obstante, en ambos casos el O precede al V, pero el orden SVO, VOS y VSO resultaría muy forzado (Cerrón Palomino 2008, 184). Entre ellos, SVO evidencia más la transferencia de la sintaxis castellana.

En el quechua las funciones gramaticales se marcan con caso morfológico mediante morfemas que se sufijan a la base léxica tanto de nombres como de verbos. Los sufijos productivos son flexionales y también formantes derivativos. Mediante sufijos junto a las bases léxicas se marcan no solo las funciones gramaticales, sino también otros fenómenos funcionales de la cláusula verbal: la topicalización, el aspecto, la temporalidad y el modo.

El español difiere del quechua en los procedimientos morfológicos. Mientras que el quechua se vale, preferentemente, de sufijos para indexar las funciones de las categorías gramaticales; el español, dada las variaciones morfológicas de sus categorías léxicas, vincula sus marcas de caso a fenómenos de concordancia y el uso de preposiciones para indicar las funciones de los sustantivos como argumentos. En tal sentido, en español, la preposición como núcleo de su sintagma precede a su complemento en un orden fijo: a diferencia del quechua, el núcleo precede a su complemento. Así, también los efectos de referencialidad de los argumentos son producidos por la presencia/ausencia de determinantes tales como artículos, pronombre $u$ otros cuantificadores que preceden al nombre (como núcleos del Sintagma Determinante SD). Marcas que en el quechua son diferentes; en este caso, se utilizan demostrativos o cuantificadores, que preceden al nombre, mientras que los posesivos son sufijos, como la marca de número.

Esta breve descripción tipológica, que no agota las diferencias entre ambas lenguas, intenta delimitar algunos de los problemas que plantea el contacto quechua-español. A pesar de ello, el hablante resuelve mediante su competencia la compatibilidad entre ambos sistemas al insertar ítems léxicos de una lengua flexiva, como el español, en el sistema quechua, lengua aglutinante, cuyas reglas morfosintácticas pertenecen a parámetros tipológicos muy diferentes.

\subsection{Especificaciones metodológicas}

El corpus fue recogido mediante entrevistas libres a diferentes vernáculohablantes de ambos sexos en diferentes contextos. En algunos casos se trató de entrevistas programadas, mientras que en otros se realizaron en sus espacios laborales, como en el caso de verdulerías o de la asistente de Educación Intercultural Bilingüe en Rawson. El corpus incluye conversaciones sostenidas entre hablantes vernáculos y permite apreciar las competencias lingüísticas que estos hablantes bilingües ponen en juego al realizar transferencias entre lenguas. 
Una de las entrevistas fue realizada por una maestra quechuahablante a una par étnica. Se entrevistó a personas cuyas edades oscilan entre veintitrés y sesenta años. Gran parte del corpus fue desgrabado y traducido con ayuda de dos hablantes de quechua, mientras que los textos breves fueron realizados por uno de los autores, siempre con el asesoramiento, cuando era necesario, del vernáculohablante. Este trabajo no incluye análisis de morfemas del quechua, sino traducción de los fragmentos de discursos vernáculos, ya que se trata de compatibilizar un enfoque sociolingüístico del contacto que intentará establecer alguna vinculación con concepciones teóricas más formales, provenientes de una mirada generativa del lenguaje.

\subsection{Entrevistados}

El corpus discursivo en lengua quechua ha sido recogido mediante grabación digital en diferentes localidades. Se ha entrevistado a femeninos y masculinos procedentes de diferentes lugares de Bolivia. La distribución geográfica de la toma de datos es la siguiente:

\begin{tabular}{ll}
\hline Localidad & Persona entrevistada \\
\hline Comodoro Rivadavia & PEG (masc), VTG (fem), MA (fem), CRV (fem) \\
Caleta Olivia & LT (fem), LTo (fem) \\
Puerto Madryn & AL (fem) \\
Esquel & MQ (masc) \\
\hline
\end{tabular}

\section{Marco teórico}

Toda lengua o variedad en contacto con otra(s) implica afectación mutua, primero a nivel individual y, en segundo lugar, a nivel de la comunidad lingüística. $\mathrm{Al}$ respecto Bynon señala que: “... toda ósmosis de material lingüístico, a través de las fronteras de las lenguas, es el resultado de cierto grado de bilingüismo por parte de quienes hacen la transferencia" (Bynon 1981, 288). El contacto lingüístico implica una serie de fenómenos propios que incluye la transferencia de elementos de una lengua a otra; por ejemplo, préstamos léxicos, cambio de código, calcos, introducción de fonemas, influencia en la sintaxis, etc.

En este trabajo se asume que la aparición de esta serie de fenómenos de contacto es factible en función de la existencia de un dispositivo mental presente en la facultad del lenguaje humano, que permite generar enunciados en cualquier lengua natural. La facultad del lenguaje, en tanto que órgano, posibilita la comprensión y producción de material lingüístico de manera computarizada mediante principios universales. Además, dicho dispositivo supone un conjunto finito de parámetros que regulan su selección (Chomsky 1988). Mientras los principios son características universales innatas e idénticas para todas las lenguas, los parámetros representan las distintas formas en que las lenguas se diferencian. Un parámetro muy conocido y que permite la comparación tipológica quechua-español es el parámetro del núcleo, que controla la posición del núcleo con respecto al complemento (Chomsky 1988a). Esto representa una diferencia paramétrica entre ambas lenguas: en español el núcleo precede al complemento, mientras que en quechua el complemento lo precede.

En el marco de las propuestas generativistas de Principios y Parámetros, el dispositivo lingüístico consta de un sistema cognitivo que almacena y 
computa información. Hay dos sistemas de salida o actuación: uno conceptual-intencional (C-I), que interactúa con el sistema cognitivo a través de la interfaz de la Forma Lógica (FL) y otro articulatorio-perceptual (A-P), que lo hace a través de la interfaz de la Forma Fonológica (FF) (Chomsky 1988b). Una vez que el hablante ha parametrizado la gramática interna de dos lenguas, y en función de sus necesidades comunicativas con el contexto social, puede combinar elementos de ambos sistemas. El sistema cognitivo le permite seleccionar un ítem léxico de una lengua fuente y por medio del sistema computacional ensamblarlo en secuencias gramaticales según los parámetros fijados de su lengua materna. Gracias a la independencia de los sistemas de salida, FL lee los rasgos o propiedades distintivas del significado del ítem léxico y la interfaz de FF lee las propiedades del sonido, mediante un proceso interno a la manera de un cómputo mental. Es el hablante en su selección el que encuentra las posibilidades de transferencias entre las diferencias paramétricas de las lenguas resolviendo los problemas del ensamble computacional que le implica cada ítem léxico.

Para retomar el enfoque sociológico: el término transferencias alude a préstamos léxicos para algunos autores, pero, para otros, se refiere a la presencia de elementos de un sistema lingüístico en otro, durante un mismo acto de habla, que va más allá del simple préstamo. Granda define a este concepto como "la totalidad de los fenómenos de modificación de estructuras originados por el influjo de una lengua sobre otra, en contacto con ella" (Granda 1996, 65).

Para Almeida transferencia implica todos los procesos que modifican la estructura de una lengua por influencia de la estructura de otra (Almeida 1999, 205). Pero el préstamo léxico no es la única presencia de una lengua o variedad en otra, como lo señalan Poplack y Sankoff, quienes enumeran una serie de procesos que permiten la ocurrencia de elementos de dos sistemas lingüísticos en un mismo discurso, tales como "cambio de código, préstamos a nivel comunidad e individuo, adquisición incompleta de la lengua, interferencia, entre otros" (Poplack y Sankoff 1987, 1174) ${ }^{1}$.

Estos mismos autores afirman que los hablantes bilingües apelan a estrategias para salvar dificultades que llevarían a la agramaticalidad, y enumeran cuatro estrategias que normalmente ocurren en estas circunstancias (Poplacky Sankoff 1987, 1175):

a) Cambio de código fluido. Es la alternancia de lenguas dentro de una misma oración en los límites sintácticos, donde no ocurriría incompatibilidad con ninguna de ambas gramáticas.

b) Cambio de código marcado. Es el que se produce atrayendo la atención del interlocutor para indicar esta alternancia de lenguas.

c) Préstamos momentáneos. Son aquellos no adoptados por la comunidad lingüística, de modo que no tienen integración social y dependen solo de la elección del hablante bilingüe.

d) Inserción de constituyentes. Consiste en insertar un constituyente en una oración en un punto apropiado, a diferencia del cambio de código, no está regido por el orden sintáctico.

Partiendo de la clasificación de Poplack y Sankoff (1987), arriba citada, en este artículo se consideran solo préstamos, transferencias sintácticas y cambio de código fluido.
1. Original: "code-switching, borrowing on the community and individual levels, incomplete language acquisition, interference, among others" (Poplacky Sankoff 1987, 1174). 


\subsection{El préstamo lingüístico}

En este apartado se hace una breve introducción al concepto de préstamo lingüístico, teniendo en cuenta los diferentes enfoques, la motivación que los origina y los grados de integración que definen el tipo.

El estudio de la ocurrencia de lexemas de una lengua en cadenas de emisión de otras y su intento de clasificación ha originado una vasta terminología, a veces sinónimos, cuasi-sinónimos o directamente definen conceptos diferentes. Como lo señala Hipperdinger:

La diversidad de enfoques que convergen sobre este campo, no obstante, ha resultado en un escaso consenso entre los especialistas sobre numerosas cuestiones, así como en una llamativa proliferación terminológica (Hipperdinger 2001, 11).

Weinreich (1953), pionero en los estudios de contacto de lenguas, llama transferencias a la inserción de unidades léxicas de una lengua en discursos de otra, y aclara que estas no se limitan solo a hablantes bilingües, sino que también pueden ocurrir en hablantes de una lengua que han adoptado el uso de lexemas de otra.

\subsubsection{Enfoques}

Gómez Cápuz (1998) presenta una síntesis de enfoques y tipologías de clasificación y concepto del préstamo, que a continuación se resume:

a) Desde el enfoque onomasiológico, tradicionalmente se abordaba el préstamo desde un enfoque onomasiológico e histórico-cultural inserto en la "tradición europea", que remonta sus orígenes hacia finales del siglo XIX (Gómez Cápuz 1998, 130-131). Los diferentes autores que se ocuparon del tema generaron una prolífica taxonomía con rótulos a veces equivalentes y otras no. Se ocuparon de justificar la necesidad y/o la legitimidad de los préstamos léxicos.

b) Desde el enfoque normativista, se cuestiona la legitimidad del préstamo dependiendo del grado de necesidad que justifique la transferencia como tal. Normalmente establecían "préstamo necesario" y "préstamo innecesario". Asimismo, admitía "préstamos técnicos" vs. "préstamos no técnicos".

c) Desde el enfoque socio-lingüístico, a partir de los estudios de Hope (1971), se considera al préstamo desde una perspectiva sociolingüística. Se parte de una valoración de las motivaciones para tomar el préstamo y se lo deja de considerar como un "barbarismo" o “extranjerismo". Este enfoque tiene en cuenta los siguientes rasgos:(i) motivaciones psíquicas, afectivas o expresivas que llevan a usar el préstamo; (ii) motivaciones lingüísticas, tales como brevedad, matices semánticos, etc.; (iii) su consideración como neologismo.

Este enfoque ha sido profundizado por Goddard (1969, 1980), mientras que Bookless (1982) propone una nueva dicotomía ("préstamos únicos" y "préstamos sinónimos"), y finalmente por Pratt (1980).

Gómez Capuz (1999, 265-66) analiza la teoría de Hope y establece como razones de existencia del préstamo las siguientes:

a) Inexistencia de sinonimia total entre la transferencia y su equivalente en la lengua meta. 
b) Rechazo de la hipótesis de las casillas vacías y explicitación de que los recursos léxicos pueden ser defectivos o inaceptables. Ese autor afirma que hay un nicho ecológico en el cual sería deseable la existencia de un término más eficaz, pero nunca hay una casilla léxica vacía;.

c) Factores lingüísticos y extralingüísticos con relación a la motivación del préstamo: (i) factores lingüísticos extrínsecos e intrínsecos, tales como innovaciones tecnológicas y culturales e ineficacia comparativa de ciertos términos nativos, respectivamente; (ii) factores extra-lingüísticos de tipo social, socio-psicológico, de distinción de clase, argótico y materiales.

\subsubsection{Motivación del préstamo}

En cuanto a las motivaciones del préstamo, Appel y Muysken (1996) las subordinan a las funciones del lenguaje y establecen las siguientes:

a) Función referencial, que implica la necesidad de nombres para objetos y conceptos nuevos, y supone la resolución de una homonimia patológica, pues opera una reestructuración de campos semánticos poco diferenciados.

b) Función expresiva y directiva, que implica la necesidad constante de sinónimos.

\subsubsection{Grado de integración de las transferencias}

Por otro lado, en el caso del préstamo hay que tener en cuenta su integración en la lengua meta. Este aspecto resulta muy discutido y a partir de él se considera si los préstamos son parte de la lengua meta o si aún pertenecen a la lengua de origen. Los factores tenidos en cuenta para definir si una transferencia es préstamo o cambio de código han sido el grado de integración social y la adaptación estructural, rasgos en los que Haugen (1973) y Lance (1975) coinciden. Por su parte, Gumperz y Hernández-Chávez (1975) tienen en cuenta la integración social aun cuando no haya adaptación estructural, en cambio Hasselmo (1970) considera que todo elemento estructuralmente adaptado está integrado y que si no está adaptado es cambio de código. Por otro lado, Berk-Seligson (1986) considera que las unidades léxicas no adaptadas estructuralmente no pueden ser instancias de cambio de código cuando no tienen equivalentes en uso en la lengua meta.

Lastra (1992, 175-189), siguiendo a Weinreich (1953), presenta la dicotomía "préstamos permanentes" y "préstamos momentáneos", y establece como criterios para caracterizar al primer tipo: (i) frecuencia de su uso; (ii) desplazamiento del sinónimo equivalente en la lengua receptora; (ii) integración morfológica y sintáctica; y (iv) aceptabilidad. No obstante, Lastra aclara que estos criterios no siempre funcionan.

En este trabajo se entiende por préstamos integrados a aquellos que se adaptan a la fonología y a la morfología de la lengua meta, especialmente observables con los fonemas que no tienen correlato y que se integran al sistema morfológico de la lengua meta, tomando morfemas derivativos o flexivos.

Por su parte, Hipperdinger señala que "en cada subsistema de las variedades lingüísticas involucradas en el contacto puede contemplarse una escala 
de transferencias posibles, que va de las de menor a las de mayor integración social" (Hipperdinger 2001, 14). La autora considera "interferencias" a las del primer tipo y "préstamos" a las del segundo tipo.

\subsection{Los préstamos en el quechua}

Las transferencias léxicas del castellano en el quechua son altamente frecuentes y se deben a diferentes causas, tales como (i) olvido, momentáneo o no; (ii) mayor grado de integración de los préstamos en la comunidad hablante; y (iii) mayor grado de integración de los préstamos en un idiolecto particular.

\subsection{Cambio de código}

El cambio de código, como lo señala Hudson, es una consecuencia inevitable del bilingüismo y afirma que "cualquiera que habla más de una lengua elige entre ellas de acuerdo con las circunstancias" $(2000,51)^{2}$. De este modo, el cambio de código no resulta caprichoso ni arbitrario, sino que el propio locutor decide cuándo hacer el cambio. Este mismo autor reconoce dos tipos:

a) Cambio de código situacional ("situational code-mixing"). El cambio coincide con el cambio de situaciones externas (Hudson 2000, 52).

b) Mezcla de códigos ("code-mixing o conversational code-mixing"). En este caso el cambio se da porque cambia la situación por sí misma o porque la lengua cambia, pero también puede operarse el cambio dependiendo del tema (Hudson 2000, 53).

Romaine (2000) señala que el cambio de código siempre está, esto se ha observado en vernáculohablantes que emiten juicios valorativos sobre la praxis discursiva de pares, tipificándolo de habla impura. Contrariamente, Appel y Muysken afirman lo siguiente:

la alternancia [de códigos] puede resultar útil para la función referencial, porque a veces implica falta de conocimiento de una lengua o falta de facilidad en una lengua para tratar un tema concreto [...] De tal modo que debemos considerar que toda alternancia inducida por un tema tratado le resultará útil a la función referencial de la lengua (Appel y Muysken 1996, 177).

Cambra señala que "el hecho de cambiar de lengua no supone una deficiencia, sino una habilidad complementaria” (Cambra 1993, 7).

\subsubsection{Perspectiva de análisis de este trabajo}

En este artículo se considera este fenómeno no como una deficiencia o falta de competencia en la lengua vernácula, sino como una habilidad del bilingüe para combinar ambas lenguas, sin caer en la agramaticalidad, y bajo ningún punto es un rasgo estigmatizante, sino una muestra de su competencia en ambos sistemas.

\section{Análisis del corpus}

En los parágrafos siguientes se consideran los diferentes tipos de transferencias léxicas del español en el quechua ${ }^{3}$.
2. “Anyone who speaks more than one language chooses between them according to circumstances" (Hudson 2000, 51).
3. Grafías y fonemas del quechua. El grafemario utilizado es el llamado "Alfabeto panquechua", que se utilizaría para las diferentes variedades: a, vocal baja central no redondeada /a/; ch, consonante africada alveolo-palatal sorda /te/; chh, consonante africada alveolo-palatal sorda aspirada /tch/; ch', consonante africada alveolo-palatal sorda glotalizada $/ \mathrm{tc}^{2} / ;$ i, vocal alta anterior no redondeada /i, [i], [e]; k, consonante oclusiva velar sorda /k/; $\mathrm{kh}$, consonante oclusiva velar sorda aspirada /kh/; k', consonante oclusiva velar sorda glotalizada $/ k^{2} /$; l, consonante lateral interdental sonora /l/; II, consonante lateral palatal sonora //; m, consonante nasal bilabial sonora $/ \mathrm{m} /$; $\mathrm{n}$, consonante nasal alveolar sonora $/ \mathrm{n} /$; $\tilde{n}$, consonante nasal palatal sonora $/ \mathrm{n} /$; $\mathrm{p}$, consonante oclusiva bilabial sorda /p/; ph, consonante oclusiva bilabial sorda aspirada / $\mathrm{p}^{\mathrm{h}} /$; p', consonante oclusiva bilabial sorda glotalizada /p?/; , consonante fricativa retrofleja sonora //; sh, consonante fricativa palatal sorda / $/ \mathrm{f}$ t t, consonante oclusiva dental sorda /t/; th, consonante oclusiva dental sorda aspirada /th/; t', consonante oclusiva dental sorda glotalizada / $\mathrm{t}^{2} / \mathrm{u}$, vocal alta posterior redondeada /u, [u], [o]; w, semiconsonante labiovelar sonora /w/; y, semiconsonante fricativa palatal sonora $/ \mathrm{j}$. 


\subsection{Préstamos y transferencias}

\subsubsection{Préstamos integrados}

\section{(a) Préstamos adaptados fonológica y/o morfológicamente}

Entre los préstamos adaptados fonológicamente en las variedades habladas en Bolivia, tal como se desprende del corpus de estudio, se pueden citar los mencionados a continuación: (i) parlay 'hablar', desplazó al vocablo rimay, que se ha resemantizado como 'discutir' (parlar es un préstamo del occitano que aparece en el Arcipestre de Hita y derivados en Berceo; en el siglo XVII se registra parlotear en Gracián (Corominas y Pascual 2010, 4:436)); (ii) yusulpayki 'gracias, te agradezco' (lit. dios se lo pague $>$ *(di) yus se lo pa(gue) > yusulpa- 'agradecer').

\section{(b) Préstamos no adaptados fonológicamente}

Los de este tipo son los más comunes, ya que se incorporaron al quechua desde los comienzos del contacto entre ambas lenguas, en el siglo XVI. Frente a la aparición de referentes nuevos para el contexto sociocultural del quechua (imperio incaico) se incorporaron lexemas españoles para nuevos conceptos introducidos por la llegada de los conquistadores a América, como escuela, castellano, dios, virgen, santo, etc. En otros casos se optó por la creación de neologismos a partir de los recursos de la lengua, por ejemplo, cantero, carbonero, panadero, herrero, etc. Si bien en algunos casos hay correlato de fonemas, no coinciden en la fonotaxis o en las realizaciones alofónicas, ya que se trata de sistemas lingüísticos diferentes. Estas transferencias no han desplazado sinónimos vernáculos y se hacen necesarios porque designan referentes nuevos, y no hay integración fonológica total, dado el correlato de los fonemas implicados.

Si bien algunos préstamos, como escuela, podrían reemplazarse por un equivalente, el neologismo quechua yachachinawasi, creado mediante los procesos morfológicos de la lengua vernácula, forma frecuente en círculos intelectuales que buscan un purismo lingüístico, la transferencia es lo más común. Posiblemente por razones de comodidad y de economía el préstamo es más frecuente en los hablantes migrantes, dado el correlato de los fonemas implicados, tanto en quechua como en español. De aquí en adelante se consideran y analizan los datos del corpus recogido entre quechuahablantes.

Un caso representativo es la incorporación léxica de "escuela" en el discurso de vernáculohablantes:

(1) Boliviapi mana mamayqa yachachiwakchukayku, escuelapi kani, cuarto kamalla karqanimatemàticas y lengua (LT, fem. Caleta Olivia).

'En Bolivia mi mamá no nos enseñaba, en la escuela, estaba hasta cuarto con matemáticas y lengua'.

(2) Noqanchis pertenecenchis chay inka puebloman, ajina qichuapiboliviapiqaescuelaspi, segundariapi yachachinku obligatorio, si o si yachananku tiyan Chay escuelaspi yachachiqtunku kosachukama? (LT, fem. Caleta Olivia).

'Nosotros pertenecemos al pueblo inca, así, en Bolivia el quechua es obligatorio en la escuela, en el secundario, sí o sí tienen que saber. En las escuelas (de aquí) si se enseñara sería bueno'. 
En (1) y (2) se observan formas verbales de yacha-'saber' y yachachi'enseñar', con las que potencialmente se podrían crear neologismos, como 'escuela', pero el venáculohablante prefiere la transferencia, que incorpora morfológicamente al tomar el sufijo locativo -pi, escuelapi 'en la escuela'. Asimismo, en otro fragmento se utiliza pesquerapi 'en la pesquera', aunque existe la raíz challwa-'pescar' se prefiere la transferencia. Este procedimiento o estrategia que el hablante utiliza resuelve la inserción del préstamo en el sistema morfosintáctico del quechua indexando su función por medio del sufijo.

(3) kutichipuyki maldicionniykita (MQ, masc. Esquel). 'Te devuelvo tu maldición'.

En (3) se observa la transferencia léxica 'maldición', seguida del sufijo posesivo -niyki 'tu' y la marca de objeto directo - $t a$. En este ejemplo se puede observar que por medio del sufijo -niyki la sintaxis del quechua marca la referencialidad del argumento. Este tipo de integración del préstamo es producto de la habilidad del hablante, en términos de su gramática interna, que con procedimientos morfosintácticos de su lengua inserta ítems léxicos según sus necesidades expresivas en secuencias gramaticales.

El ejemplo de (3) destaca uno de los aspectos más interesante del contacto quechua-español: bajo qué condiciones los hablantes alteran el orden canónico de la oración en quechua. Al respecto, por el momento solo se está en condiciones de describir el fenómeno en función de la fijación de dos valores opuestos para el mismo parámetro: la posición del núcleo verbal con respecto a su complemento objeto como una alternancia en el uso que puede ser explicada como un fenómeno de contacto. Esta variación se produce en particular con el núcleo verbal y sus modificadores. En tal sentido, y dentro de la teoría de Principios y Parámetros, esta alternancia del habla informal es una variación del parámetro del núcleo que da lugar a una variedad dialectal, entendida como una lengua distinta que como hablantes "sabemos cuándo usarla, una en un sitio otra en otro. Ahora bien, cada una de estas lenguas supone una distribución de interruptores [parámetros] distinta" (Chomsky 1988, 152). En tal sentido, el estudio más pormenorizado de dichas ocurrencias excede los límites de este trabajo.

Por otro lado, la oración completa sigue la sintaxis del español, en la que el verbo se antepone al objeto, a diferencia del quechua, en el que se pospone.

(4) Kay wasiypi qhechuatapuni parlayku (LT, fem. Caleta Olivia). 'En mi casa hablamos puro quechua'.

El préstamo ha generado dos entradas léxicas para precisar una diferencia de significado ya que no hay sinonimia total entre parlay, con el sentido de competencia lingüística, y rimay 'discutir', como intercambio de opinión.

Por otro lado, coincidiendo con Lastra (1992), se puede ver que estas transferencias son de uso frecuente, que están integradas morfológica y sintácticamente y que gozan de aceptabilidad, pero en la práctica no han desplazado ningún sinónimo vernáculo, que es uno de los rasgos que Lastra considera para la integración de los préstamos. 


\subsubsection{Préstamos no integrados}

En los parágrafos siguientes se presentan ejemplos de este tipo de préstamo según clases de palabras y también morfemas gramaticales.

\section{(a) Verbos}

La formación del infinitivo quechua, que se indexa con el morfema $-y$, se combina con las transferencias del español; por ejemplo, trabajar $+-y$ : trabajay. Luego en la conjugación toma todos los morfemas flexivos: trabajani 'trabajo', trabajanki 'vos trabajás', trabajarqani 'había trabajado', trabajasaq 'trabajaré', etc. En estos casos se observa que se insertan en la posición sintáctica de la lengua meta. En esta oración se incorpora el sufijo de flexión -ni, marca de $1 \stackrel{0}{\circ}$ singular.

(5) Nuqa pesquerapi trabajani (LT, fem. Caleta Olivia). 'Yo trabajaba en la pesquera'.

Entre los verbos más frecuentes incorporados como préstamos no integrados se pueden citar los siguientes: baylay ('bailar'), consiqiy ('conseguir'), creey ('creer'), ekstrañay ('extrañar'), enfermay ('enfermarse'), enseñay ('enseñar'), entiendiy endiendey ('entender'), fallay ('fallar'), kalentachiy ('calentar'), kambiay ('cambiar'), nacey ('nacer'), nestay ('necesitar'), pertenecey ('pertenecer'), tokay ('tocar un instrumento'), trabajay ('trabajar'), utilizay ('utilizar').

Si bien estas transferencias no forman parte del léxico quechua, en su uso se conjugan según la morfología de la lengua meta. Estos préstamos alternan con sus equivalentes vernáculos, como se desprende del corpus estudiado:

(6) Nuqasutiyqa Adela López. Kaypi, ElBpi trabajasani, Ilank'asani, y así, tawa, tawataquraqniyoq, quraqniyoq trabajasani kayEIBpi [...] Madrinmanta viajaqhamuni kayman, Rawsoman, a trabajaq, Ilank'aq hamuni (AL, fem. Pto. Madryn).

'Me llamo Adela López. Aquí, en la ElB estoy trabajando, estoy trabajando, y así, cuatro, más de cuatro años, más de (cuatro) años estoy trabajando en esta EIB [...] Viajo de Madryn para acá, a Rawson, vengo a trabajar, a trabajar'.

En el fragmento precedente se ve la autocorrección de la vernáculohablante, cuando usa la transferencia e inmediatamente el lexema quechua equivalente, llank'asani 'estoy trabajando', llank'aq 'para trabajar'. Esto demuestra que la hablante tiene conciencia lingüística acerca del préstamo realizado. Se han tratado de encontrar los lexemas quechuas equivalentes a las transferencias, y los hablantes consultados (V, fem. y PEG masc., Comodoro Rivadavia) han utilizado verbos con semas parecidos o incluso construcciones perifrásticas:

\begin{tabular}{ll}
\hline entiendiy endiendey 'entender' & hapiy 'agarrar, captar'; yachay 'saber' \\
\hline ertenecey 'pertenecer' & -paq, sufijo casual dativo \\
\hline consiqiy 'conseguir' & tariy 'buscar' \\
\hline kambiay 'cambiar' & waqtakay 'ser otro' \\
\hline
\end{tabular}

Este tipo de préstamos muestra un mayor grado de complejidad y ellos dependen, por un lado, del olvido momentáneo o tal vez permanente, hecho 
relacionado con la situación sociolingüística, el español como lengua franca y de contacto social aún más empleado dentro del contexto étnico. Aún cuando se considera que este tipo de transferencias no forma parte del sistema vernáculo, por el alto grado de frecuencia y de ocurrencia en contextos similares, habría que plantearse si serían parte del sistema de diferentes idiolectos, como lo sugieren Poplack y Sankoff (1987), arriba citados, quienes hablan de préstamos a nivel de individuo. Pero también habría que considerarlos, a través de una lente diacrónica, como cambios surgidos por el contacto tan prolongado.

El siguiente fragmento ejemplifica las ocurrencias de préstamos:

(7) -A. ¿Imapi trabajanki? (LT, fem. Caleta Olivia).

-B. Nuqa pesquerap itrabajani (LTo, fem. Caleta Olivia).

-A. ¿Siempre trabajarqanki?

-B. Siempre trabajarqani pesquerapi, Conarpescapi Puertopiqa trece añosta, kaypi kaykuntan trabajasani dos añosta.

-A. ‘¿En qué trabajas?’

-B. 'Yo trabajo en la pesquera'.

-A. '¿Siempre trabajaste?’

-B. 'Siempre trabajé en la pesquera, Conarpesca en el Puerto 13 (trece) años, aquí trabajé 2 (dos) años'.

En el corpus analizado, ambos hablantes nativos del quechua, solo por una cuestión de preferencia, integran morfológicamente el préstamo trabajay pero en el caso de B (primera ocurrencia) el verbo está pospuesto, según la sintaxis quechua; en B (segunda ocurrencia) el verbo no se encuentra al final sino que se antepone a sus modificadores, en un orden más similar al español.

\section{(b) Nombres}

(8) ¿Ima edadpiyachakurqankicastellanuta parlayta, kay kaqmanta pacha yachanki? (LTo, fem. Caleta Olivia).

‘¿Desde qué edad aprendiste a hablar español, desde cuándo sabés?’.

Los siguientes préstamos están integrados solo morfológicamente: el nombre edad se ubica antepuesto al verbo en el constituyente que incluye el pronombre interrogativo ima 'qué'; por otro lado, el nombre presenta un sufijo - pi, marca de caso locativo. Del mismo modo castellanuta, complemento objeto del verbo parlay se antepone según el orden SOV propio del quechua. Morfológicamente se le incorpora el sufijo - $t a$, marca de acusativo.

\section{(c) Adjetivos}

(9) ¿castellanotaparlasankiri perfecto, le costachuparlankiotumpa más o menos? (LTo, fem. Caleta Olivia).

‘Castellano hablas perfecto o te cuesta hablar más o menos?’.

En este ejemplo, más allá de los préstamos no integrados fonológicamente, el enunciado mantiene el orden del español, ya que el verbo parlay 'hablar' se antepone al adjetivo y por lo tanto no va en posición final. En la segunda 
oración, el primer constituyente es el verbo español 'costar' al que se añade el sufijo -chu, marca de interrogación.

(10) Castellanuta tumpa chakchuta parlani, mana correctotachu (LT, fem. Caleta Olivia).

'El castellano lo hablo un poco mezclado, no correctamente'.

En este caso el adjetivo se adverbializa al tomar el sufijo -ta, que precede al sufijo de negación -chu.

(d) Adverbios

(11) Casi 2002 jampuni Boliviamanta (AL, fem. Pto. Madryn).

'Casi en el 2002 vine de Bolivia'.

El adverbio casi se inserta en la oración y el verbo no va al final, según el orden prototípico del quechua.

(12) Siempre qakarqayku La Platapi, La Platamanta jampuyku Puerto Madrynman. La Platapi kayku dos añosta, Puerto Madrynpinkta kayku huq watata (LT, fem. Caleta Olivia). 'Siempre estábamos en La Plata, de La Plata nos vinimos a Puerto Madryn. En La Plata estuvimos dos años, En Puerto Madryn estamos desde hace un año'.

El adverbio siempre, antepuesto al verbo, va marcado por el sufijo qa, que señala al constituyente topicalizado. Por otra parte, los nombres propios que son topónimos también se encuentran integrados en sus posiciones y de acuerdo a sus funciones sintácticas mediante los sufijos -pi para la posición del locativo como en La Platapi. Mientras que, en el segundo sintagma, el sufijo -manta, en La Platamanta indica al complemento locativo del verbo con un sentido de lugar de origen, pero el sufijo -man en Puerto Madrynman funciona como circunstancial de lugar con sentido de meta.

Como lo sugieren Poplack y Sankoff (1987), los préstamos no integrados serían a nivel de individuo, por lo tanto, ocurren en los idiolectos particulares, pero también habría que considerarlos de manera diacrónica como cambios surgidos por el contacto fluido, sobre todo en el caso de algunos de ellos como son "entonces", "siempre", "pero", "a veces", "porque".

\section{(e) Sufijos derivativos}

También se observa la ocurrencia del sufijo derivativo nominal "-ero/ -era":

(13) qhechalerata japichun (PEG, masc. Comodoro Rivadavia). 'que le agarre una cagadera'.

En este ejemplo ocurre el morfema "-era" sufijado a la base verbal qhecha 'tener diarrea', originando cagadera. Asimismo, calca la expresión castellana "agarrarle algo".

La ocurrencia del sufijo agentivo "-(d)or":

(14) mana kanchu qhaqudores llaqtaykipi? (PEG, masc. Comodoro Rivadavia). ‘¿no hay masajistas en tu ciudad?’. 
El morfema "-dor" sufijado a la base verbal qhaquy 'masajear' genera qhaqudor "masajista'. Asimismo, el hablante añade el sufijo "-es", marca de plural, lo que constituye, a su vez, una unidad calcada de la morfología del español.

En estos casos las transferencias de estos elementos de significado funcional son también sufijos del español; es decir, en esta ocasión, ambas lenguas comparten el mismo procedimiento morfológico. Tal vez sean préstamos motivados por la necesidad de nombres para conceptos nuevos (función referencial según Appel y Muysken (1996)). Sin embargo, esto deja traslucir la conciencia de los hablantes bilingües que identifican la semenjaza de procedimientos morfosintácticos entre ambas lenguas. Se valen así de un recurso del español accesible en su contexto social para la creación de vocablos mediante un procedimiento que dominan como hablantes del quechua: la derivación mediante sufijos.

\subsubsection{Transferencia en el nivel sintáctico}

El contacto intenso y prolongado entre diferentes lenguas lleva a la convergencia, como la presentó Gumperz (1971). En la aldea multlingüe de Kupwar (Maharashtra, India), este autor observó una asombrosa intertraductibilidad entre tres lenguas, que se reducía básicamente a diferencias léxicas, ya que la sintaxis y muchas categorías gramaticales tenían un mismo patrón. En este caso, el quechua está en contacto con el español desde el siglo XVI, tiempo suficiente para acercar ambas sintaxis y permitir la transferencia del suprasistema:

(15) nuqaman gustawan kutiy jaqayman (MA, fem. Comodoro Rivadavia). 'a mí me gusta volver allá'.

En este ejemplo, no solo el orden es el del español (SVO), sino que se calcan las estructuras de esta lengua, nuqaman 'a mí', utilizando el caso ilativo 'hacia' y la forma verbal gustawan, que imita la forma castellana 'me gusta'. En el corpus se observan abundantes casos de transferencia de la sintaxis del castellano, como se aprecia también en (2) con orden SVComp, en (3) VO y en (16) Vcomp.

\subsection{Cambio de código}

El siguiente fragmento ilustra el cambio de código que el hablante hace al introducir oraciones en español en su discurso en quechua:

(16) Qichuataastawanmanejani, mamaywan, hermanusninwan,

qichuamantapuniparlariykunanqaplantapipis, casi mayoría paisanos kayku,

llampaqtaqkarqanku Argentinos plantapi, castellanostaparlaqkaykunoqayku.

Kunanqa hasta kayku paisanos, así que, que se llama, napiqari, que se llama, controlankuchaypispaisanallataq, siempre qichuamana puniniwaykuwasaulektaruwachiq. Llampaqtan Argentinos karqankuchaypitaqri puro castellanotaparlaqkayku, kunanricasi mayoríaqichuapipuniparlariyku. (LT, fem. Caleta Olivia).

'Quechua lo manejo más, con mi mamá, mis hermanos, quechua es lo que más hablamos en la planta, casi la mayoría son paisanos, trabajaban argentinos en la planta, teníamos que hablar castellano. Ahora hay paisanos, así que, que se llama, le dicen, que se llama, la que controla también es paisana, siempre en quechua nos dice ‘hagan bien'. Antes había más argentinos, ahí teníamos que hablar exclusivamente en castellano, ahora, casi la mayoría hablamos en quechua'. 
Las instancias de cambio de código evidencian la influencia del quechua en el español utilizado, en 'casi [la] mayoría' la ausencia del artículo como categoría funcional de la que prescinde el quechua. Es interesante notar, también, la mezcla de código en 'puro castellanuta' aunque esta última categoría presenta su marca de caso, con el sufijo -ta, que indexa objeto, el orden de palabra AN respeta el orden del español; mientras que una expresión similar qichuapipuniparlariyku respeta el orden NA, propio del quechua. En tal sentido, el cambio de código no altera la estructura morfosintáctica.

(17) Yachachinipís pero mana lloksinchupalabrana, parlaymunan, pero kakutaparlanwan, manakikintaparlanchu "haqaytapasamuay" Niní "patamusaqichu" niwan, mana lloksinchu "eso pásame yo te dije" Nini, eso entiende más fácil. Y en quechua es otra cosa no entienden tanto, qosawanqaqichuapipuniparlanku (LT, fem. Caleta Olivia).

'Le enseño pero no le sale la palabra, quiere hablar pero raro habla, no habla igual, "pásame eso" le digo y dice y no se le entiende, no le sale y le digo "pásame eso te dije" y eso entiende más fácil. Y en quechua es otra cosa no entiende tanto, con mi marido hablamos en quechua siempre'.

En el fragmento precedente se observa la ocurrencia de estilo directo, ya que el hablante se cita a sí mismo hablando en español. La cita es retomada como tópico a través del deíctico 'eso', lo que constituye una explicación de lo que estaba diciendo. En dicha instancia ocurre el cambio de código, a continuación de la cita textual, al hacer una explicación sobre el intento de hablar quechua por parte de su hijo. Estas instancias de cambio de código ejemplifican las ya citadas afirmaciones de Cambra $(1993,7)$.

\section{Conclusiones}

El contacto entre ambos sistemas lingüísticos ha permitido una serie de fenómenos propios de esta situación e inevitables que, dado la vitalidad del vernáculo y el continuo e intenso contacto, ha llevado a un incremento constante de las transferencias del español en el quechua, hecho del que algunos hablantes tienen conciencia y por ende dicen "hablamos mezclado". Pero no por ello disminuyen las transferencias, sino al contrario, como se ha podido ver en los ejemplos incluidos en este trabajo.

Los lexemas incorporados pasan a ser parte del sistema y, por lo tanto, son utilizados por la comunidad lingüística. Estas transferencias están adaptadas fonológica y morfológicamente. Si bien no tienen adaptación fonológica, especialmente en el caso de fonemas que tienen correlato, toman los morfemas quechuas pertinentes para poder ser utilizados, principalmente, en el caso de los verbos. Las transferencias no incorporadas al sistema serían préstamos a nivel idiolectal y son comprensibles para otros hablantes debido al bilingüismo de la comunidad quechua. A pesar de que estos préstamos se pueden considerar a nivel idiolectal, serían factibles de ser utilizados por otros hablantes, dada la competencia en ambas lenguas que ellos poseen.

Los hablantes bilingües toman diversas categorías: no solo nombres, sino también verbos, adjetivos, adverbios y conjunciones, y las integran mediantes procedimientos morfosintácticos. En el caso de las categorías léxicas, las integran por medio de la sufijación indexando sus funciones en la cláusula. Los hispanismos más frecuentes son verbos, sustantivos y adjetivos, hecho que no excluye unidades de clases cerradas, como conjunciones, subordinantes y coordinantes. Asimismo, se observa la transferencia de 
morfemas ligados que se incorporan a bases léxicas quechuas, hecho que muestra la profundidad del contacto. Estas transferencias ocurren, obviamente, en registros no formales, ya que el corpus de estudio corresponde a la lengua hablada.

Respecto a la sintaxis, si bien hay influencia del español y las oraciones que siguen el orden de esta lengua son abundantes, muchas oraciones siguen el orden prototípico del quechua. Esta cuestión merece más atención de la que aquí se le ha dedicado y queda abierta para otros estudios más pormenorizados.

Con esta breve descripción y análisis de fenómenos se pretende resaltar las habilidades que los hablantes bilingües desarrollan para resolver las diferencias que enfrentan en el dominio de su competencia gramatical entre ambas lenguas, cuando por circunstancias de contacto realizan una transferencia de una lengua a otra. Así, por ejemplo, la transferencia de un nombre les plantea problemas que se relacionan con el caso, o su referencialidad, o si el constituyente se encuentra alcanzado por los significados funcionales de la cláusula verbal; cuestiones que los hablantes deben resolver, en principio, de manera individual.

Si bien este análisis no permite afirmar más que estas transferencias, lo descripto forma parte del idiolecto de los hablantes como variedad de su lengua materna, usada en su ámbito comunitario, familiar, laboral y sociocultural en Patagonia. Revisten un alto grado de competencia en el dominio de ambas gramáticas y, posiblemente, constituyen variedades utilizadas por la comunidad migrante para satisfacer funciones referenciales y expresivas de su actividad discursiva que significan un reconocimiento de los esfuerzos de adaptación e integración a su nuevo destino social realizado por esa comunidad migrante. 


\section{Q Bibliografía}

" Almeida, Manuel. 1999. Sociolingüística. Santa Cruz de Tenerife: Universidad de La Laguna.

»Appel, René y Pieter Muysken. 1996. Bilingüismo y contacto de lenguas. Barcelona: Ariel.

》 Berk-Seligson, Susan. 1986. "Linguistic constraints on intrasentential code-switching: a study of Spanish/Hebrew bilingualism”. Language in Society 15.3: 313-348.

" Bookless, Thomas C. 1982. "Towards a semantic description of English loan-words into Spanish". Quinquereme V, 2: 170-185.

» Bynon, Theodora. 1981. Lingüística histórica. Traducción de José L. Melena. Madrid: Gredos.

" Cambra, Margarida. 1993. "Canvis de llengua i discurs a classe de francés llengua estrangera a l'ensenyament primari". Tesis de doctorado, Universidad de Barcelona.

"Cerrón Palomino, Rodolfo. 2008. Quechumara: Estructuras paralelas del quechua y del aimara. La Paz: UMSS, PROEIB Andes, Plural Editores.

"Chomsky, Noam. 1970. Aspectos de la teoría de la sintaxis. Madrid: Aguilar.

"Chomsky, Noam. 1988a. El lenguaje y los problemas del conocimiento: Conferencias de Managua I. Madrid: Visor.

"Chomsky, Noam. 1988b. La nueva sintaxis: teoría de la rección y ligamento. Barcelona: Paidós.

» Corominas, Joan y José A. Pascual. 2010. Diccionario crítico etimológico castellano e hispánico. Madrid: Gredos.

" Goddard, Keith A. 1969. "Loan-words and lexical borrowing in Romance". Revue de Linguistique Romane 33: 337-348.

" Goddard, Keith A. 1980. "Loan-words into Spanish: A reappraisal”. Bulletin of Hispanic Studies LVII: 1-16.

" Gómez Capuz, Juan. 1998. "El préstamo lingüístico: Concepto, problemas y métodos". Anexo XXIX de la Revista Cuadernos de Filología. Valencia: Universitat de València.

" Granda, Germán de. 1996. "Interferencia y convergencia sintácticas e isogramatismo amplio en el español paraguayo". International Journal of the Sociology of Language 117: 63-80.

» Gumperz, John J. 1971. Language in social groups. Standford University Press.

» Gumperz, John J. y Eduardo Hernández-Chávez. 1975. “Cognitive aspects of bilingual communication". En El lenguaje de los chicanos: Regional and social characteristics of language used by Mexican Americans, editado por Eduardo Hernández-Chávez et al., 154-163. Arlington: Center of Applied Linguistics.

» Hasselmo, Nils. 1970. "Code switching and modes of speaking". En Texas studies in bilingualism: Spanish, French, German, Czeck, Polish, Sorbian and Norwegian in the Southwest, editado por Glenn Gilbert, 179-210. Berlin: Walter De Gruyter.

》 Haugen, Einar. 1973. "Bilingualism, language-contact and immigrant of languages in the United States: A research report 1956-1970". En Current Trends in Linguistic, volume 10: Linguistics in North America, editado por Thomas A. Sebeok, 505-91. De Haag: Mouton. 
» Hipperdinger, Yolanda. 2001. Integración y adaptación de transferencias léxicas: Contribución al estudio del contacto lingüístico en español bonaerense. Bahía Blanca: EdiUNS.

» Hope, Thomas E. 1971. Lexical borrowing in the Romance language: A critical study of Italianisms in French and Gallicisms in Italian from 1100 to 1900. Oxford: Basil Blackwell.

» Hudson, Richard A. 2000 [1981]. Sociolingüística. Barcelona: Anagrama.

» Lance, Donald. 1975. "Spanish-English code switching”. En El lenguaje de los chicanos: Regional and social characteristics of language used by Mexican Americans, editado por Eduardo Hernández-Chávez et al., 138-153. Arlington: Center of Applied Linguistics.

» Lastra, Yolanda. 1992. Sociolingüística para hispanoamericanos: Una introducción. México DF: El Colegio de México.

» Poplack, Shana y David Sankoff. 1987. “Code-switching”. En Sociolinguistcs/Soziolinguistik, editado por Ulrich Ammon et al., II: 1174-80. Nueva York: Walter de Gruyter.

» Pratt, Chris. 1980. El anglicismo en el español peninsular contemporáneo. Madrid: Gredos.

» Romaine, Suzanne. 200o. Language in society: An introduction to sociolinguistics. Oxford: OUP.

»Quiroz Villarroel, Alfredo. 200o. Gramática quechua. La Paz: Ministerio de Educación, Cultura y Deporte.

» Weinreich, Uriel. 1970 [1953]. Languages in contact. Findings and problems. De Haag: Mouton. 